\section{Endoscopic extraction of Fasciolopsis buski}

A 20-year-old, married, primigravida presented with a history of epigastric discomfort, nausea, and vomiting for 3 days. On the day of admission, she gave a history of vomiting up a $3-4-\mathrm{cm}$, pearshaped, reddish-brown worm, which moved for a few seconds after it was vomited and then died. On further enquiry, the patient gave a history of consumption of vegetables that had been washed in pond water.

Upper gastrointestinal endoscopy revealed a reddish-brown, flat worm stuck in the second part of the duodenum (Figure 1, Video 1) The worm was extracted with the help of biopsy forceps under endoscopic guidance. The worm was $4 \mathrm{~cm}$ long, $2 \mathrm{~cm}$ wide, and about $0.25 \mathrm{~cm}$ thick, reddish-brown, flattened anteroposteriorly, and pear-shaped, with no prominent or obvious cephalic cone, and resembled the fasciolopsis buski fluke (Figure 2). Histopathological examination of the specimen confirmed it as F. buski. Routine investigations revealed a normal peripheral-smear eosinophil count. Stool examination did not reveal any F. buski eggs. The patient was treated with praziquantel $25 \mathrm{mg} / \mathrm{kg}$ as a single dose. The patient was asymptomatic and doing well on followup.

To our knowledge, this is the first report describing and illustrating the endoscopic removal of $F$. buski from the duodenum using forceps. Fasciolopsis and other food-borne trematode infections are included in the list of important helminthiases that have a great impact on human development. Current changes in global weather patterns appear to be increasingly affecting snail-borne helmin-

\section{Video 1}

Upper gastrointestinal endoscopy, showing live, mobile F. buski in the second part of the duodenum. The worm was extracted using biopsy forceps.

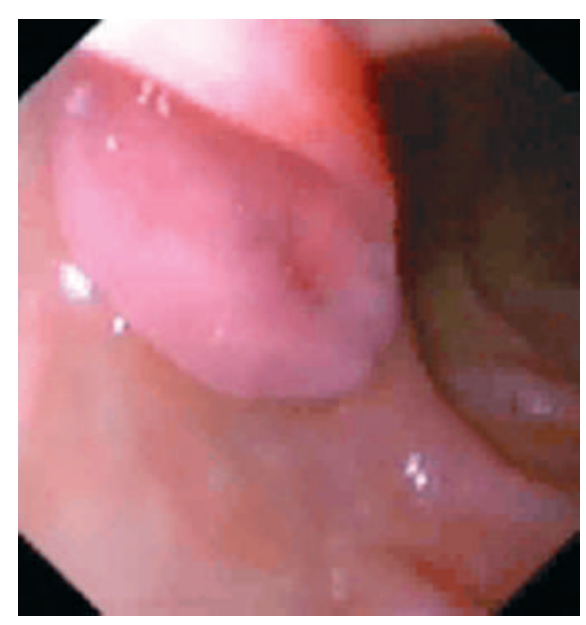

Figure 1 Endoscopic view of fasciolopsis bus$k i$ stuck in the second part of the duodenum.

thiases, which are strongly dependent on environmental factors. Fasciolopsis is a good example of a parasitic disease that is emerging (or re-emerging) in many countries as a consequence of changes in both environmental and human factors $[1,2]$. Unfortunately, despite control programs, $F$. buski still remains a public health problem in endemic areas and in areas where it was once thought to have been controlled $[3,4]$.

Though the disease is seen predominantly in south-east Asia, the endoscopic image of $F$. buski we have included here may be of interest to the rest of the world because of immigration, globalization, and the increased frequency of intercontinental travel.

Endoscopy_UCTN_Code_CCL_1AB_2AZ_3AZ

Endoscopy_UCTN_Code_TTT_1AO_2AN

M. Murugesh' ${ }^{1}$, S. Veerendra', S. Madhu', S. Kude', A. D. Amrapurkar ${ }^{2}$, U. U. Rathi', P. M. Rathi ${ }^{1}$

${ }^{1}$ Department of Gastroenterology and Hepatology, BYL Nair Charitable Hospital and TN Medical College, Mumbai, India

2 Department of Pathology, BYL Nair Charitable Hospital and TN Medical College, Mumbai, India.

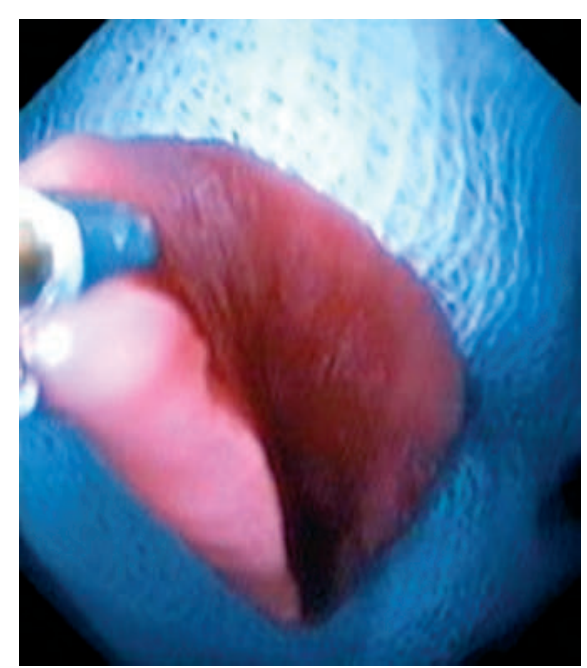

Figure 2 The endoscopically extracted $F$. buski worm was $4 \mathrm{~cm}$ long, $2 \mathrm{~cm}$ wide, and about $0.25 \mathrm{~cm}$ thick.

\section{References}

${ }^{1}$ Mas-Coma S, Bargues MD, Valero MA. Fascioliasis and other plant-borne trematode zoonoses. Int J Parasitol 2005; 35: $1255-$ 1278

2 Bhatti HS, Malla N, Mahajan RC, Sehgal R. fasciolopsiasis - a re-emerging infection in Azamgarh (Uttar Pradesh). Indian J Pathol Microbiol 2000; 43: 73 - 76

${ }^{3}$ World Health Organization. Foodborne trematode infections. Bull WHO 1995a ; 73: 397-399

${ }^{4}$ World Health Organization. Control of foodborne trematode infections. WHO Tech Rep Ser 1995b ; 849: 1 - 157

\section{Corresponding author}

\section{P. M. Rathi, MD}

Department of Gastroenterology and Hepatology

BYL Charitable Nair Hospital and TN Medical College

Dr. AL Nair Road

Mumbai Central

Mumbai

Maharastra 400008

India

Fax: $\quad$ +91-22-23021168

Email: rathipm@hotmail.com

DOI: $10.1055 / \mathrm{s}-2006-945154$ 